\title{
Prueba de impulso cefálico
}

\author{
Head impulse test
}

Hayo A. Breinbauer $\mathbf{K}^{1}$, José Luis Anabalón B'1.

\section{RESUMEN}

Introducción: Dentro del estudio de la función del equilibrio, la prueba de impulso cefálico representa una herramienta rápida, sencilla y de fácil interpretación, que explora la indemnidad del reflejo óculo-vestibular. Este examen ha cobrado relevancia reciente como complemento a la prueba calórica en diversos contextos y no ha sido explorado en nuestro país.

Objetivo: Explorar sensibilidad y especificidad de esta prueba en nuestro contexto local, junto con contribuir a la difusión de una herramienta de creciente relevancia en otoneurología.

Material y método: Estudio prospectivo, ciego, de evaluación de test diagnóstico. En una muestra de pacientes consultando por vértigo agudo se realizó prueba de impulso cefálico al momento de la consulta inicial, y al momento de realizar prueba funcional de VIII par.

Resultados: Se evaluaron 52 pacientes, $44 \%$ de ellos con disfunción vestibular según prueba calórica clásica como patrón de oro. La sensibilidad de la prueba de impulso cefálico fue de $47,6 \%$ y su especificidad de $83,9 \%$, con un valor predictivo positivo y negativo de $66,7 \%$ y $70,3 \%$. El acuerdo del examen entre dos evaluadores independientes fue alto (Kappa $=0,84$ ).

Discusiones: A pesar de una baja sensibilidad por sí sola, la prueba de impulso cuenta con una alta especificidad y un valor predictivo positivo razonable. Es además altamente reproducible, realizable en menos de un minuto y no genera náuseas u otros síntomas en el paciente. Consideramos este examen como un complemento al estudio de la función vestibular, con implicaciones prácticas en el inicio precoz de terapia y con importantes potencialidades en diversos ámbitos de la otoneurología.

Palabras clave: Reflejo óculo-vestibular, pruebas vestibulares funcionales.

\begin{abstract}
Introduction: In balance function evaluation, head impulse test is a fast, simple and easy to interpret test, which explores the vestibulo-ocular reflex. Having gained in the last decade growing importance as a complement to traditional caloric test, this test hasn't been explored in our context yet.

Aim: To assess sensitivity and specificity of this test in our context, along with contributing to the spread of an increasingly important tool in otoneurology.
\end{abstract}

\footnotetext{
${ }^{1}$ Médico. Departamento Otorrinolaringología, Pontificia Universidad Católica de Chile.

${ }^{2}$ Tecnólogo Médico. Departamento Otorrinolaringología, Pontificia Universidad Católica de Chile.
} 
Material and method: A prospective, blinded, diagnostic test assessment. In a sample of patients consulting for acute vertigo, head impulse test was performed at the time of initial consultation, and at the time of VIII nerve functional testing.

Results: We evaluated 52 patients, $44 \%$ with vestibular dysfunction as shown in caloric test results as gold standard. Sensitivity of the head impulse test was $47.6 \%$, its specificity $83.9 \%$, with positive and negative predictive value of $66.7 \%$ and $70.3 \%$ respectively. Agreement between two independent examiners was high $(K a p p a=0.84)$.

Discussion: Despite its low sensitivity, impulse test showed a high specificity and a reasonable positive predictive value. It was also highly reproductible, and generates no nausea or other symptoms in the patient. We consider this test as a complement to vestibular function evaluation, with clinical applicationssupportingearly onset of therapy. We believe this test to withhold significant potential in various otoneurological developing applications.

Key words: Vestibulo-ocular Reflex, Vestibular function tests.

\section{INTRODUCCIÓN}

La función del balance y el equilibrio en el ser humano, resulta de la integración neurosensorial central de tres sistemas: visual, propioceptivo y vestibular ${ }^{1}$. La disfunción periférica de este último se manifiesta clásicamente como un síndrome vertiginoso, siendo una de las consultas más frecuentes en otorrinolaringología².

Anatómica y funcionalmente, podemos clasificar la función vestibular periférica en dos partes: las estructuras dependientes del nervio vestibular superior (canales semicirculares superior y lateral, utrículo y una pequeña porción del sáculo), y las dependientes del nervio vestibular inferior (canal semicircular posterior y la mayor parte del sáculo).

La evaluación de la función del nervio vestibular inferior es aún motivo de debate, y dentro de las pruebas disponibles actualmente destaca la prueba calórica mínima y, con mayor validación a nivel internacional, los potenciales evocados miogénicos vestibulares (VEMP) $)^{3,4}$.

Por otro lado la prueba calórica es el referente histórico y vigente de evaluación de la función del nervio vestibular superior, ya sea a ojo desnudo 0 bajo apoyo de video-óculo-nistagmografía (esta última es considerada patrón de oro para este parámetro $)^{3,5}$. Esta prueba se basa en la estimulación 0 inhibición de la función tónica, principalmente del canal semicircular lateral, al inducir un movimiento de endolinfa mediante un cambio en su densidad. Este cambio sería provocado por el cambio de temperatura que se produce por la instilación de agua fría o caliente en el conducto auditivo externo. Así, se produce una estimulación 0 inhibición vestibular ipsilateral aprovechando una característica física de la endolinfa, que como cualquier líquido, se mueve en un eje vertical respecto a la fuerza de gravedad frente a cambios en su densidad (característica que depende de la temperatura del líquido).

En la práctica se ha estandarizado instilar el conducto auditivo externo con agua a dos temperaturas distintas $\left(17^{\circ} \mathrm{C}\right.$ y $\left.44^{\circ} \mathrm{C}\right)$, siendo un efecto secundario habitual de la prueba gatillar una intensa sensación vertiginosa, la cual forma parte de la interpretación del examen.

La prueba de impulso cefálico representa una alternativa en la evaluación de la función vestibular, la cual ha cobrado gran relevancia en la literatura en los últimos años. Consiste en una prueba simple, sencilla y rápida, ideada originalmente dentro del examen físico otoneurológico básico en la entrevista inicial del paciente. Sin embargo, este examen se está perfilando como un complemento formal a la prueba calórica clásica, al evaluar la función vestibular en una forma mucho más fisiológica ${ }^{6,7}$.

Esta prueba (que puede ser encontrada en la literatura como "head impulse test" o "head thrust test') está basada en el reflejo óculo-vestibular. Este consiste en la compensación de movimientos cefálicos por parte de los músculos extrínsecos oculares, de tal forma que la mirada pueda permanecer fija en un objeto pese a los movimientos de la cabeza, por bruscos y rápidos que éstos sean. 
En la Figura 1 vemos un ejemplo de este reflejo. Al ser girada bruscamente la cabeza del paciente por el examinador (solicitando mantener la mirada fija en un punto lejano y estático), se genera la ilusión que los ojos "no se mueven" manteniendo la fijación ocular en el objeto. Lo que realmente ocurre es que al girar la cabeza del paciente en un plano horizontal, se está estimulando la función del canal semicircular lateral ipsilateral a la dirección del giro (en este caso el canal lateral derecho, dado el giro hacia la derecha). El aparato vestibular estimulado, activa a su vez a los músculos extrínsecos de ambos ojos (recto medial en el ojo ipsilateral y recto lateral en el ojo contralateral, inhibiendo a su vez los músculos antagonistas.) para contraerse en directa proporción al giro cefálico, moviendo ambos ojos en forma compensatoria, logrando mantener la vista fija en el objeto. La descripción previa explica los movimientos horizontales en base al estímulo del canal horizontal, pero el reflejo vestíbulo-ocular ocurre en todas las dirección del espacio, ocupando distintas combinaciones de canales semicirculares y músculos oculomotores ${ }^{8}$.

En un paciente con una disfunción vestibular este reflejo se encuentra alterado y disminuido. Supongamos una paresia vestibular de las estructuras dependientes del nervio vestibular superior derecho (Figura 2). Al girar el examinador la cabeza del paciente hacia la derecha, el canal horizontal derecho no es estimulado (justamente porque está alterada su función y no responde, o responde en menor medida a la maniobra). Al no ser estimulado, no se genera un impulso nervioso hacia los músculos extrínsecos oculares, los que se quedan inmóviles respecto a la cabeza, desviando la mirada del objeto en el que estaban fijos (Figura 2b). Esta aparente "desviación de los ojos" manifiesta la positividad de la prueba de impulso cefálico y representa una hipofunción vestibular. La sustancia reticular, al igual que con el nistagmo, corrige la posición ocular a la "esperada" (fija en el objeto) gatillando una sácada correctiva que lleva los ojos nuevamente hacia el objeto (Figura 2c).

Existen diferentes formas de realizar y evaluar la prueba de impulso cefálico. Un aspecto importante es realizar los giros cefálicos con la cabeza inclinada $30^{\circ}$ hacia abajo. De esta forma se alinean los canales horizontales con el eje del giro de la maniobra, dado que la posición anatómica natural de estos canales semicirculares es justamente con una leve inclinación hacia arriba (Figura 3). Está técnica mejora la sensibilidad y especificidad del test ${ }^{9}$.

El impulso cefálico fue concebido originalmente para ser realizado a ojo desnudo. Bajo esta modalidad su sensibilidad (inclinando cabeza a $30^{\circ}$ ) se encuentra entre $55 \%$ y $72 \%$, y su especificidad entre $78 \%$ y $83 \%$. En un intento de mejorar estos valores se han implementado distintas técnicas para detectar retrasos más sutiles en el reflejo vestíbulo-ocular.

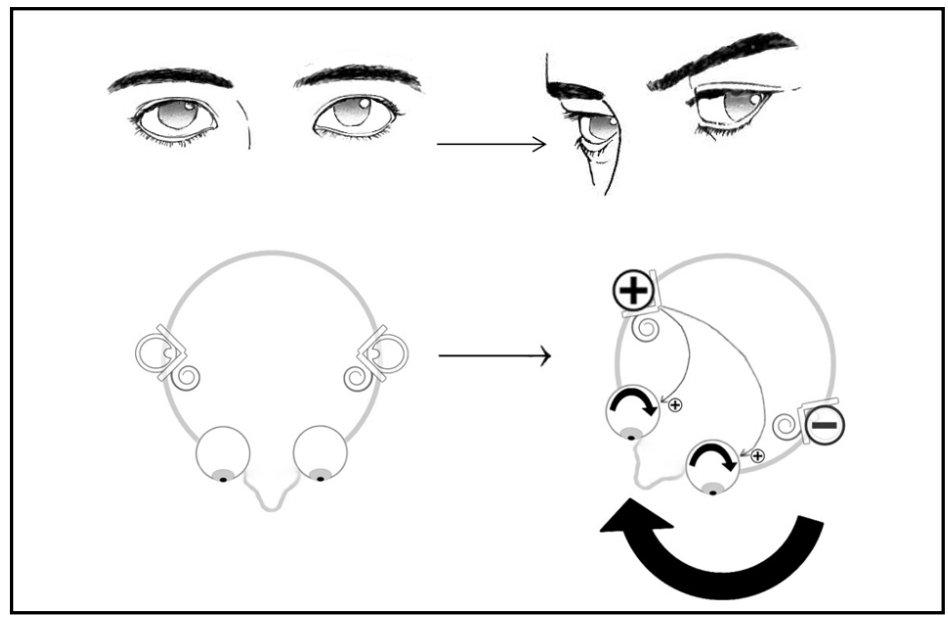

Figura 1. Reflejo óculo-vestibular normal. 
Figura 2. Reflejo óculo-vestibular alterado, con una paresia vestibular derecha.

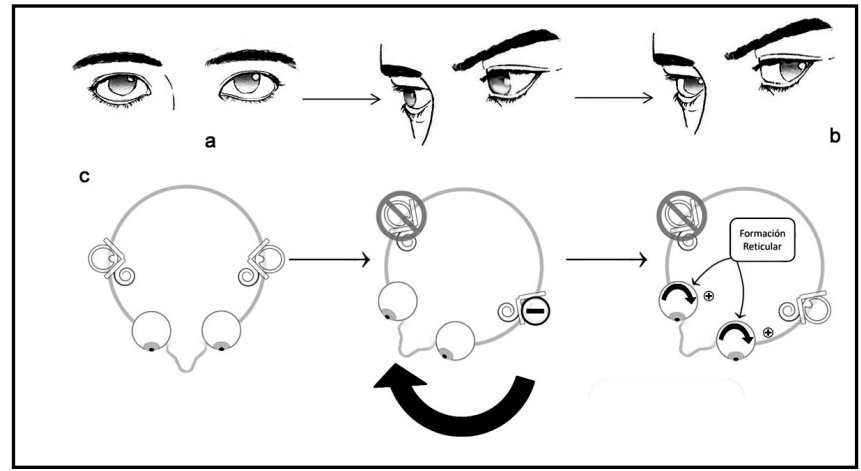

Hasta 2009 el patrón de oro para esta prueba era el seguimiento tridimensional con bobinas corneales, examen relativamente invasivo donde se instala una bobina de cobre a modo de lente de contacto en el paciente, la que puede ser seguida punto a punto con sensores electromagnéticos ${ }^{10}$. Asociado a un sensor fijo en la cabeza del paciente se pueden registrar simultáneamente la velocidad angular de giro cefálico (movimiento provocado por el examinador) y la velocidad angular del giro ocular (movimiento producto del reflejo vestíbulo-ocular hacia contralateral y compensatorio, registrado por las bobinas cocleares). La relación entre ambas velocidades se conoce como la ganancia del reflejo vestibular-ocular. Valores cercanos a 1 representan un reflejo eficiente, mientras que si se acercan a 0 representa un reflejo disminuido o patológico. Con esta tecnología la sensibilidad de la prueba rodea el $84 \%$, con una especificidad de $91 \%{ }^{10}$.

Recientemente ha aparecido una evaluación de la prueba de impulso cefálico con videooculografía de alta velocidad. Esta herramienta noinvasiva logró una sensibilidad y especificidad de $100 \%$ en detectar alteraciones del reflejo vestíbuloocular respecto al uso de bobinas corneales, situándose como un posible nuevo patrón de oro en este test ${ }^{11}$.

La utilidad de esta prueba es mayor cercana a una crisis 0 evento vestibular agudo. Se describe una recuperación de la ganancia del reflejo vestíbulo-ocular en el tiempo incluso a dos semanas de un episodio de neuronitis vestibular. Se postula que por procesos de compensación semejantes a la rehabilitación vestibular, el aparato vestibular no afectado supliría la función necesaria para mantener un seguimiento ocular, ${ }^{6,7}$.

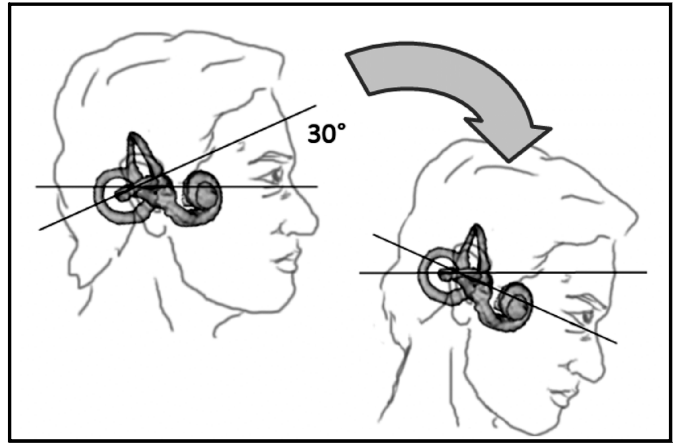

Figura 3. Al inclinar la cabeza en $30^{\circ}$ se alinean los canales horizontales con un verdadero plano horizontal al realizar los giros cefálicos.

En nuestro país no se ha evaluado el uso de esta prueba diagnóstica, por lo que el presente estudio pretende determinar su sensibilidad y especificidad en una muestra chilena, buscando además difundir una herramienta potencialmente útil en otoneurología.

\section{MATERIAL Y MÉTODO}

Se realizó un estudio prospectivo, ciego, de evaluación de test diagnóstico.

Considerando una potencia estadística de $80 \%$, un error alfa de $5 \%$ y en base a frecuencias de positividad del test y de presencia de paresia vestibular en patrón de referencia en estudios previos y semejantes $^{3,7,7,11}$ se calculó un tamaño muestral necesario de 46 pacientes para lograr encontrar valores confiables de la prueba en estudio.

La muestra se escogió por criterios de factibilidad (no probabilística) y consistió en pa- 
cientes atendidos por residentes participando en el estudio en el Departamento de Otorrinolaringología de la Pontificia Universidad Católica de Chile entre abril y septiembre de 2010, que hayan consultado por inestabilidad 0 vértigo agudo (de más de 20 minutos de duración en su crisis inicial, como criterio de inclusión) y que hayan tenido indicación de estudio de VIII par por su médico tratante. Se consideró como criterio de exclusión presentar nistagmo espontáneo al momento de la consulta 0 de la realización del VIII par, o contar con historia de enfermedad vestibular conocida previa.

Se realizó una evaluación piloto en 10 pacientes, que sirvió de adiestramiento en la prueba para los residentes, tecnólogos médicos y estudiantes de tecnología médica que participaron en el estudio.

Completada esta etapa, se reclutaron, previo consentimiento informado, a pacientes que consultaran con los residentes participantes. En esta primera entrevista se realizaba anamnesis, examen otoneurológico básico (que incluía pesquisa de nistagmo espontáneo sin lentes de Frenzel, examen de pares craneanos y pruebas cerebelosas), además de la prueba de impulso cefálico por parte del residente.

A continuación se solicitó examen de VIII par, en cuya instancia dos tecnólogos médicos (0 alumno de tecnología médica) realizaban prueba calórica clásica y prueba de impulso cefálico. Ambos tecnólogos se mantuvieron ciegos entre sí respecto al resultado de ambas pruebas.

En nuestro centro la prueba calórica es utilizada con apoyo de video-oculonistagmografía dependiendo de las posibilidades económicas y preferencias del paciente, lo que se realizó según procedimiento habitual. Además, en aquellos casos que la prueba calórica resulta simétrica, pero la sensación vertiginosa experimentada durante ella se asemeja a la sintomatología por la que el paciente consulta, es nuestro protocolo habitual sospechar alteración del nervio vestibular inferior, por lo que se solicita complementar el estudio con una prueba calórica mínima.

Se analizan los resultados considerando sólo la prueba calórica clásica como patrón de oro (con o sin video-oculonistagmografía), y por separado considerando la prueba calórica mínima como complemento.

Se realizaron cálculos de sensibilidad y especificidad con software SPSS 18.0, en base a las evaluaciones al momento del VIII par por ambos tecnólogos médicos (no considerando para este análisis la evaluación inicial por el residente, para evitar sesgo por cambios en el pacientes producidos por la diferencia de tiempo entre la consulta inicial y el momento de realización del VIII par).

Se comparó además nivel de acuerdo, mediante índice Kappa, entre la evaluación de la prueba de impulso cefálico realizada por el residente al momento de la entrevista inicial y la evaluación por el tecnólogo al momento del VIII par, buscando confiabilidad interevaluador.

\section{RESULTADOS}

Se evaluaron 52 pacientes, 9 de ellos con apoyo de video-oculonistagmografia y 43 con prueba calórica clásica a ojo desnudo. En 2 casos se solicitó además prueba calórica mínima como complemento. La edad promedio de la muestra fue de 49,5 años (desviación estándar 15,1 años), donde 56,8\% fueron mujeres. El 40,4\% de la muestra presentó una paresia vestibular en la prueba calórica clásica, mientras que 3,8\% presentó alteración exclusiva en la prueba calórica mínima. Si consideramos prueba calórica mínima, $44,2 \%$ de la muestra presentó paresia vestibular, dado que este examen resultó positivo en ambos casos solicitados.

No hubo otros hallazgos dentro de la muestra evaluada, como vértigo postural paroxístico benigno dentro de los pacientes incorporados al estudio.

El acuerdo entre residente y tecnólogo alcanzó un acuerdo sustancial, con un valor Kappa de 0,84.

En la Tabla 1 se detallan los resultados de la prueba calórica cruzada con la prueba de impulso cefálico (en paréntesis se detallan los resultados considerando también prueba calórica mínima). La relación entre positividad del impulso cefálico y presencia de paresia vestibular es estadísticamente significativa (prueba de $\mathrm{Chi}^{2}$ con $\mathrm{p}<0,01$ ).

En base a estos datos, podemos apreciar en la Tabla 2 como la prueba de impulso cefálico alcanza una sensibilidad baja, de $47,6 \%$, pero una buena especificidad de $81,9 \%$ (52,3\% y $88,7 \%$, respectivamente si consideramos prueba calórica mínima como parte del patrón de oro). El valor predictivo positivo fue de $66,7 \%$ con un valor predictivo negativo de $70,3 \%$ (80\% y 70,3\% si consideramos prueba calórica mínima como parte del patrón de oro). 
Tabla 1. Resultados prueba impulso cefálico y prueba calórica. La prueba de impulso cefálico fue tomada en base a su realización por tecnólogo médico durante el VIII par. ()* corresponden a los valores de la tabla si se incluye la prueba calórica mínima como parte del patrón de oro

\begin{tabular}{|lcccc|}
\hline & & \multicolumn{2}{c|}{ Prueba calórica } & \\
& & Alterada & Normal & Total \\
\hline Prueba de & Alterada & $10(12)^{*}$ & $5(3)^{*}$ & 15 \\
impulso cefálico & Normal & 11 & 36 & 37 \\
& Total & 23 & 29 & 52 \\
\hline
\end{tabular}

Tabla 2. Sensibilidad y especificidad de prueba de impulso cefálico. En la columna de la izquierda se aprecian los valores de sensibilidad y especificidad si consideramos sólo la prueba calórica como patrón de oro, y en la derecha, el resultado si consideramos también la prueba calórica mínima dentro del patrón de oro

\begin{tabular}{|lcc|}
\hline Prueba de impulso cefálico & \multicolumn{2}{c|}{$\begin{array}{c}\text { Patron de Referencia } \\
\text { Prueba calórica + mínima }\end{array}$} \\
\hline Sensibilidad & Prueba calórica & $52,2 \%$ \\
Especificidad & $47,6 \%$ & $89,7 \%$ \\
Valor predictivo positivo & $83,9 \%$ & $80,0 \%$ \\
Valor predictivo negativo & $66,7 \%$ & $70,3 \%$ \\
\hline
\end{tabular}

\section{DISCUSION}

En la experiencia de este estudio, la prueba de impulso cefálico ha demostrado ser un examen de fácil aprendizaje, ejecución e interpretación para el estudio de la función vestibular, habiendo demostrado ser altamente reproducible entre distintos evaluadores (Kappa $=0,84)$.

Destacan dentro de las ventajas de esta prueba su brevedad de ejecución (sólo algunos segundos, dentro de la misma maniobra en que se puede explorar presencia de nistagmo espontáneo) y la ausencia de experimentación de vértigo, náuseas 0 mareos secundarios a su implementación.

En este trabajo hemos explorado su utilidad realizada a ojo desnudo, con una inclinación de $30^{\circ}$ hacia abajo, buscando alinear los canales semicirculares horizontales con el eje de giro cefálico. Bajo estas circunstancias hemos obtenido resultados de sensibilidad y especificidad equipa- rables a los reportados en la literatura en condiciones similares.

Mientras la sensibilidad es en general baja, la especificidad es relativamente alta. Se describe en la literatura que la positividad en esta prueba, asociada a la sospecha clínica por el otorrinolaringólogo logran valores predictivos positivos bastante altos, sobre el $85 \%^{3}$. Sin embargo esta prueba, por sí sola, logró el $66,6 \%$ de valor predictivo positivo en nuestra casuística. Así, dos de cada tres pacientes que presenten una prueba de impulso cefálico alterada (sin considerar ningún otro elemento de la anamnesis en el análisis, fuera de haber presentado una crisis de inestabilidad 0 vértigo de al menos 20 minutos de duración, que consistió en el motivo de consulta), tendrán efectivamente una alteración en su función vestibular utilizando la prueba calórica clásica como patrón de oro.

Estos parámetros mejoran si consideramos la prueba calórica mínima como parte del patrón de 
oro. Aunque el blanco central de la maniobra es estimular el canal horizontal, fisiológicamente se estarían estimulando también los canales superior e inferior. No existe evidencia publicada que asocie la prueba de impulso cefálico horizontal con potenciales evocados miogénicos vestibulares (VEMP) como estudio del compromiso del nervio vestibular inferior. Sin embargo es fisiológicamente esperable que una alteración exclusiva del nervio vestibular inferior, también pueda alterar el resultado del reflejo vestíbulo-ocular explorado en esta prueba.

Así, considerando tanto la prueba calórica clásica como la prueba calórica mínima como parámetros que reflejan alteración de la función vestibular ${ }^{4}$, podemos considerar que la prueba de impulso cefálico a ojo desnudo y con inclinación de $30^{\circ}$ tiene un valor predictivo positivo de $80 \%$.

Independiente de si consideramos la prueba calórica mínima como parte del patrón de oro, la prueba de impulso cefálico impresiona como gran complemento para detectar hipofunción vestibular en el momento de la primera consulta. Es altamente probable, que en todo paciente con sospecha de hipofunción vestibular se realice de todas formas un examen de VIII par. Sin embargo es habitual que la realización de este examen sea diferida algunos días, por disponibilidad del examen o por el uso de medicamentos al momento de la primera consulta que alteren el resultado del VIII par como benzodiacepinas 0 antivertiginosos. Esta latencia entre indicar un VIII par y conocer su resultado, puede posponer el inicio de terapias que han demostrado utilidad en disminuir el daño en la función vestibular, como el uso de corticoides sistémicos $^{12-14}$.

Así, la verdadera utilidad práctica de pruebas complementarias como el impulso cefálico, reside en un respaldo concreto al decidir el inicio temprano de terapia con corticoides. Este tratamiento, aunque no ha demostrado disminuir la duración 0 intensidad de síntomas en la crisis inicial, si parece fomentar la recuperación de función vestibular y aparentemente disminuir tanto la inestabilidad residual como la posibilidad de recurrencia ${ }^{12-15}$. A la vista de la evidencia actual, el inicio de corticoides sistémicos en altas dosis parece mandatorio en pacientes con una fuerte sospecha clínica de neuronitis vestibular que no tengan contraindica- ciones por otras patologías de base para esta terapia.

En otras aplicaciones, varios centros utilizan la medición cuantitativa del reflejo óculo-vestibular, medido mediante bobinas cocleares o más recientemente video-oculonistagmografía de alta velocidad, para evaluar la disminución aguda de la función vestibular como parámetro de éxito del tratamiento intratimpánico con gentamicina en pacientes con enfermedad de Ménière. Este contexto favorece este examen sobre el estudio habitual de VIII par, tanto por la facilidad de poder repetirlo luego de cada inyección, como por el hecho de medir hipofunción aguda justo tras la acción de gentamicina ${ }^{8,16-18}$

Será interesante revisar en el futuro, a medida que mejoren y se difundan los métodos de análisis de movimientos oculares sutiles, si el estudio del reflejo vestíbulo-ocular se instala como una alternativa razonable para el estudio de la función vestibular, donde podría alcanzar la sensibilidad y especificidad de la prueba calórica (sin el gasto de tiempo y el habitual gatillo de síntomas nauseosos propios de nuestro actual patrón de oro), y quizás incluso pudiendo aislar la función de cada canal semicircular de forma independiente en todos los posibles ejes de giro cefálico y no sólo el horizontal.

\section{BIBLIOGRAFÍA}

1. Visser J, Carpenter M, van.der.Koolj H, Vloem B. The clinical utility of posturography. Clin Neurophysiol 2008; 119(11): 2424-36.

2. Yin M, Ishikawa K, Wong W, Shibata Y. A clinical epidemiologial study in 2169 patients with vertigo. Auris Nasus Larynx 2009; 36: 30-5.

3. Wuyts F, Furman J, Vanspaumen R, VanderHeyning P. Vestibular function testing. Curr Opin Neurol 2007; 20(1): 19-24.

4. Riveros H, Callejas C, Nieme C, Gajardo P, Anabalón J, CorRea C. Importancia clínica de la prueba calórica mínima. Rev Otorrinolaringol Cir Cabeza Cuello 2007; 67: 217-21.

5. Fitzgeral G, Hallpike C. Studies in human vestibular function: II. Observation on the directional preponderance of caloric nystagmus resulting from cerebral lesions. Brain 1945; 65: $138-60$. 
6. Barti K, Lehnen N, Kohlbecher S, y c. Head impulse testing using video-oculography. Ann N Y Acad Sci 2009; 1164: 331-3.

7. Weber $\mathrm{K}, \mathrm{Aw} \mathrm{S}, \mathrm{TOdd} \mathrm{M}$, y c. Horizontal impulse test detects gentamicin vestibulotoxicity. Neurology 2009; 72(16): 1417-24.

8. Carey J, Minor L, Peng G, Della-Santina C, Cremer $P$, Haslwanter T. Changes in the three-dimensional angular vestibulo-ocular reflex following intratympanic gentamicin for Ménière's disease. J Assoc Res Otolaryngol 2002; 3(4): 430-43.

9. Shubert M, Tusa R, LE G, y c. Optimizing the sensitivity of the head thrust for identifying vestibular hypofunction. Physical Therapy 2004; 84(2): 17-25.

10. Weber K, MacDougall H, Halmagyi G, y c. Impulsive testing of semicircular-canal function using video-oculography. Ann $N \mathrm{Y}$ Acad Sci 2009; 1164: 486-91.

11. MacDougall H, Weber K, LA M, y c. The video head impulse test: diagnostic accuracy in peripheral vestibulopathy. Neurology 2009; 73(14): 1134-41.

12. Goudakos J, Markou KD, Franco-Vidal V, Vital V, Tsaligopoulos M, Darrouzet V. Corticosteroids in the Treatment of Vestibular Neuritis: A Sys- tematic Review and Meta-Analysis. Otol Neurotol 2010; 31: 183-9.

13. Shupak A, Issa A, Golz A, Kaminer M, Braverman I. Prednisone Treatment for Vestibular Neuronitis. Otol Neurotol 2008; 29: 368-74.

14. Strupp M, Zingler V, Arbusow V, Nicklas D, Maag $\mathrm{K}$, Dieterich M, et AL. Methylprednisolone, Valacyclovir or the combination for Vestibular Neuritis. N Engl J Med 2004; 351(4): 354-9.

15. Kammerlind A, Ledin T, EIB S, LM Ö. Long-term follow up after acute unilateral vestibular loss and comparison between sbjects with and without remaining symptoms. Acta Otolaryngol 2005; 125: 946-53.

16. MINoR L. Intratympanic gentamicin for control of vertigo in Meniere's disease: vestibular signs that specify completion of therapy. Am J Otol 1999; 20(2): 209-19.

17. Park H, Migliaccio A, Della-Santina C, Minor L, CAREY J. Search-coil head-thrust and caloric test in Ménière's disease. Acta Otolaryngol 2005; 125(8): 852-7.

18. Steenerson R, Hardin R, Cronin G. Gentamicin injections for Ménière disease: comparison of subjective and objective end points. Ear Nose Throat J 2008; 87(8): 452-6. 